\title{
Granulomas del encéfalo. A propósito de un caso
}

Ariel Varela Hernández¹, Félix Orellana Cortez¹, María Berenice García Gimenez², Claudio Martínez Terreu¹, Reinaldo Torres Aravena1, Patricio Herrera Astudillo', Rodolfo Muñoz Gajardo¹, Luis Lamus Aponte ${ }^{1}$

1 Neurocirujanos. Servicio de Neurocirugía. Hospital Regional de Talca. Chile.

2 Especialista en Imagenología. Hospital Regional de Talca. Chile.

Rev. Chil. Neurocirugía 44: 154-159, 2018

\section{Resumen}

Introducción: El diagnóstico de granuloma intracraneal constituye un reto clínico, sobre todo en pacientes sin antecedentes orientadores. Se presenta el caso de un paciente joven, inmunocompetente, operado por tumor cerebral y con confirmación por biopsia de tuberculoma. Se ofrece una revisión sucinta sobre el tema. Caso clínico: Paciente de 29 años, sin antecedentes patológicos, debuta con cefalea y convulsión tónico clónica generalizada. El examen físico al momento del ingreso no mostró alteraciones. La Resonancia Magnética cerebral evidenció una lesión tumoral intraaxial, frontal derecha, con gran edema perilesional y efecto de masa. La Tomografía Computarizada contrastada de tórax, abdomen y pelvis fue normal. Se realizó la resección en bloque de la lesión y la biopsia escisional confirmó un tuberculoma. Conclusiones: Para el diagnóstico de los granulomas intracraneales es esencial la sospecha clínica, teniendo en cuenta los antecedentes epidemiológicos, la detección de estados de inmunodepresión y de lesiones sistémicas concomitantes. Varios exámenes neuroimagenológicos, inmunológicos y microbiológicos son de ayuda, no obstante el estudio histopatológico de las lesiones es determinante.

Palabras clave: Granulomas intracraneales, tuberculoma intracraneal, resonancia magnética del cerebro.

\begin{abstract}
Introduction: Diagnosis of intracranial granuloma is a clinical challenge, especially in patients without pathological medical history. We present the case of a young, immunocompetent patient, operated for a brain tumor and confirmed tuberculoma by biopsy. A succinct review on the subject is offered. Clinical case: A 29-year-old patient with no pathological history, debuted with headache and generalized tonic clonic seizure. Physical examination at admission showed no alterations. Magnetic Resonance of the brain showed an intraaxial right frontal tumor, with great perilesional edema and mass effect. Contrast computed tomography of the thorax, abdomen and pelvis was normal. Bloc resection of the lesion was performed and the excisional biopsy confirmed a tuberculoma. Conclusions: Clinical suspicion is essential for the diagnosis of intracranial granulomas, taking into account the epidemiological antecedents, the detection of immunodepression states and concomitant systemic lesions. Several neuroimaging, immunological and microbiological tests are helpful, although the histopathological study of the lesions is crucial.
\end{abstract}

Key words: Intracranial granulomas, intracranial tuberculoma, MRI.

\section{Introducción}

Debido a su baja frecuencia, sobre todo en pacientes sin antecedentes de inmunodepresión, las enfermedades granulomatosas cerebrales son raramente planteadas como diagnóstico presuntivo primario en los enfermos que se presentan con tumores cerebrales.

Los granulomas se presentan como tumores no neoplásicos, encapsulados, resultado de un proceso inflamatorio crónico, donde puede o no revelarse áreas de necrosis en su interior. Dichas afecciones incluyen una gama variada de causas donde se destacan las producidas por micobacterias, hongos, parásitos e inflamatorias. Como consecuencia de su presentación, muchas veces inespecífica desde el punto de vista clínico e imagenológico, con frecuencia el diagnóstico es un reto y la confirmación descansa fundamental- 
mente en el examen histopatológico de las lesiones ${ }^{16}$.

Se presenta el caso de un paciente joven, sin inmunocompromiso diagnosticado que fue operado para la resección de un tumor cerebral, cuyo resultado histopatológico mostró un tuberculoma cerebral. Motivados por este hallazgo se ofrece una revisión sucinta de las enfermedades granulomatosas cerebrales de mayor interés en la práctica neuroquirúrgica.

\section{Caso clínico}

Paciente de 29 años de edad, sin antecedentes patológicos relevantes, normolíneo, atleta. Al ingreso narró historia de aproximadamente 2 meses de cefalea hemicraneana derecha progresiva, la cual se había agudizado en los últimos 8 días, asociada a un evento de pérdida de conciencia súbita, sin causa aparente, seguida de movimientos tónico clónicos generalizados de pocos minutos de duración y amnesia al suceso. Al examen físico general, regional y por aparatos y sistemas no se detectaron alteraciones.

Los exámenes hematológicos y hemoquímicos generales no mostraron alteraciones. VHS y PCR normales. VIH y VDRL plasmática negativos. Se practicó una Resonancia Magnética (RM) cerebral con contraste donde se detectó una lesión intraaxial, frontal derecha, con compromiso ependimario de ventrículo lateral ipsilateral, rodeada de acentuado edema que provocaba importante efecto de masa (Figura 1). Se plantearon las opciones de neoplasia de origen glial versus lesión granulomatosa. Se realizó además una tomografía computarizada (TC) contrastada de tórax, abdomen y pelvis sin alteraciones.

Se operó mediante craneotomía osteoplástica frontal derecha. Con la ayuda de la ultrasonografía transoperatoria de localizó la lesión tumoral en región frontal subcortical, la cual resultó ser encapsulada, con plano de clivaje nítido con el tejido cerebral, cuyo límite medial se encontraba en íntimo contacto con la pared del ventrículo lateral ipsilateral. Aplicando técnicas microquirúrgicas se logró la resección en bloque de la lesión sin eventualidades transoperatorias y se envió la muestra a biopsia. El período postoperatorio trascurrió sin complicaciones, no se

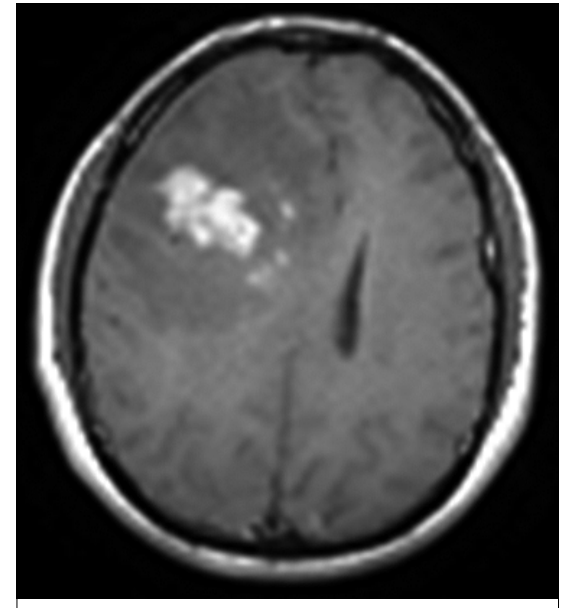

Figura 1. RM contrastada en $T_{1}$ que muestra lesión tumoral frontal derecha, subcortical, con captación de contraste e importante edema perilesional.

detectaron déficits neurológicos y se tomaron neuroimágenes de control que confirmaron la resección tumoral total, con mejoría evidente del edema cerebral perilesional (Figura 2 y 3 ).

Después de 15 días de evolución postoperatoria favorable reingresa por cuadro de fiebre moderada intermitente, cefalea bifrontal acompañada de vómitos y rigidez nucal $X$ como único hallazgo al examen físico. No obstante, el paciente se observó con buen estado general y la zona quirúrgica sin alteraciones aparentes. Se reiteran exámenes hematológicos y hemoquímicos generales, los cuales resultaron normales. PCR, VHS y procalcitonina: Normales. TC de cráneo simple: No sugerente de ninguna complicación quirúrgica.

Se practicó punción lumbar para examen de líquido cefalorraquídeo (LCR): Aspecto claro. Incoloro. Glóbulos Rojos: 25-30 x campo. Glóbulos Blancos: 4-6 x campo. Glóbulos de pus: No se observaron. Recuento de Leucocitos: $0,305 \times 10^{3} \mathrm{~mm}^{3}$. Mononucleares: $58 \%$. Polimorfonucleares: $42 \%$. Proteínas: 106,3 mg/dl. Glucosa: 22 mg/dl. ADA: 4,3 U/L. GRAM, cultivo bacteriológico y tinta china: Negativos.

El cuadro remitió después de 5 días con tratamiento sintomático. Se recibió el resultado de la biopsia que evidenció una lesión granulomatosa crónica, con zona necrotizante tipo tuberculoide. Técnicas de PAS para hongos y PCR para micobacterias negativas. Se concluye el caso como tuberculoma

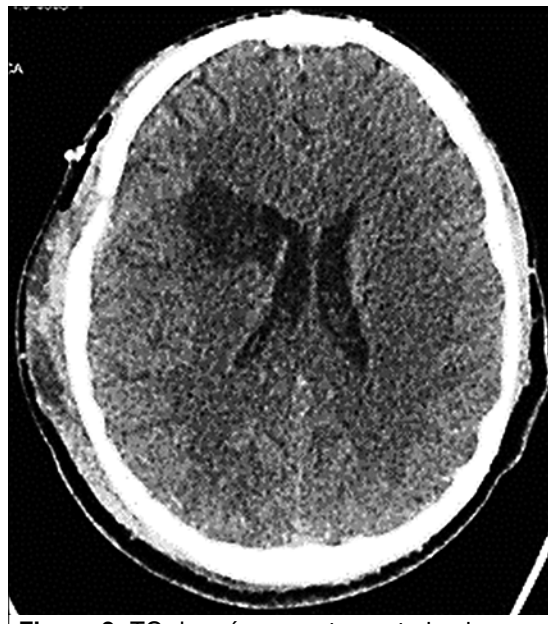

Figura 2. TC de cráneo postoperatoria sin contraste, sin evidencias de complicaciones del lecho quirúrgico, con resolución del edema perilesional y la luxación de la línea media.

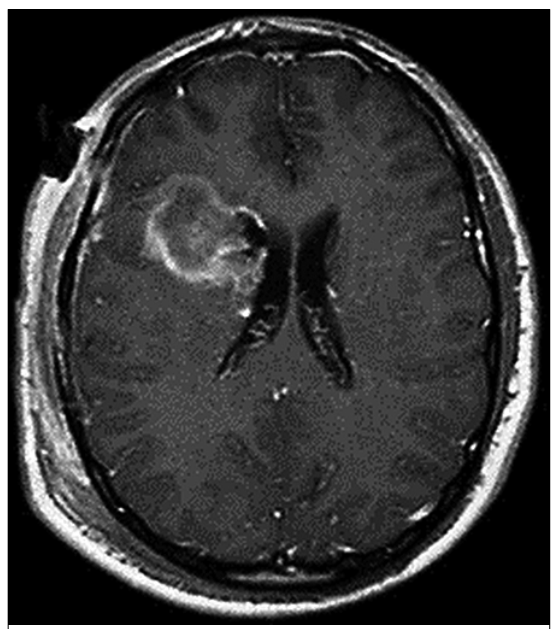

Figura 3. RM postoperatoria contrastada en $T_{1}$ que muestra resección total de la lesión, captación de contraste en los márgenes de la resección, mejoría del edema cerebral y la luxación de la línea media.

cerebral. Se interconsulta con el departamento de infectología y se decide iniciar tratamiento antituberculoso. Después de 3 meses la evolución continúa de forma favorable.

\section{Discusión}

Los tumores intracraneales constituyen un grupo heterogéneo de lesiones, frecuentes en la práctica neuroquirúrgica, donde predominan los relacionados con neoplasias primarias o metástasis del encéfalo. Los granulomas, en com- 
paración, son los protagonistas solo en un número muy reducido de individuos; por otro lado, su presentación tanto clínica como imagenológica, carece de signos patognomónicos y además, fuera del examen histopatológico, los estudios inmunológicos y microbiológicos están permeados por una sensibilidad y especificidad limitadas. Estos hechos provocan que en casos donde no se detecten antecedentes epidemiológicos de riesgo, inmunodepresión u otras lesiones sistémicas, dichos tumores no se planteen como la primera opción diagnóstica.

La formación de granulomas en el encéfalo, como resultado del contacto de un individuo con el agente causal, depende mucho más de las particularidades de la respuesta inmunológica del huésped que del agente. Los granulomas del encéfalo comprenden una gama amplia de causas: infecciosas, parasitarias o inflamatorias ${ }^{1}$, según se resumen en la Tabla 1.

En referencia a las causas infecciosas tenemos en primer lugar al micobacterium tuberculoso, el cual será comentado más adelante y el Treponema pallidum. En relación a este último, la presencia de Goma Sifilítico constituye en la actualidad una de las presentaciones más infrecuentes de neurosífilis. En estos granulomas se verifica la existencia de necrosis en examen histopatológico. Además de la recogida cuidadosa de datos que apoyen el padecimiento de sífilis primaria y secundaria, el examen con VDRL del LCR cuenta con un gran rendimiento diagnóstico ${ }^{29}$.

Varias especies de hongos han sido relacionados con la formación de granulomas intracraneales. En general se presentan como lesiones oportunistas, pero se han descrito también en pacientes inmunocompetentes; pueden alcanzar el encéfalo por continuidad o contigüidad, previa colonización de senos perinasales, o por vía hematógena como en el caso de la infección pulmonar.

Son más frecuentes los causados por Aspergirus $^{10,26}$, Blastomicosis ${ }^{24}$, Paracoccidioidomicosis $^{12}$, entre otras. Como ocurre en las otras causas infecciosas, el diagnóstico se sustenta en la titulación de anticuerpos plasmáticos hacia las diferentes especies, cultivos en medios apropiados, técnicas de amplificación y reconocimiento del DNA (PCR) y examen histopatológico; en los cuales, aplicando tinciones adecuadas, pue-

Tabla 1.

Principales causas de granulomas intracraneales

\begin{tabular}{|l|l|}
\hline Causas & Especificaciones \\
\hline Infecciosas & $\begin{array}{l}\text { *Bacterianas: Micobacterium tuberculoso, Treponema pallidum } \\
\text { *Micóticas: Aspergirus, Cándida, Histoplasma, Blastomicosis, } \\
\text { Criptococo, Paracoccidioidomicosis }\end{array}$ \\
\hline Parasitarias & $\begin{array}{l}\text { Cisticerco, Toxoplasma, Tripanosoma cruzi (Enfermedad de } \\
\text { Chagas), Amebas (Acanthamoeba spp, Balamathia madrillaris, } \\
\text { Sappinia pedata) }\end{array}$ \\
\hline Inflamatorias & $\begin{array}{l}\text { Cuerpo extraño intracraneal*, Neurosarcoidosis, Enfermedad } \\
\text { de Wegener*, Enfermedad granulomatosa crónica, Granulo- } \\
\text { matosis con poliangeítis }\end{array}$ \\
\hline
\end{tabular}

Leyenda: *Granulomas con verificación de necrosis en el examen histopatológico.

Tabla 2.

Diagnóstico diferencial de las lesiones en anillo intracraneales

\begin{tabular}{|l|l|}
\hline Causas & Tipos anatomo clínicos \\
\hline Neoplasias & $\begin{array}{l}\text { Primarias: Glioma de alto grado de malignidad, Linfo- } \\
\text { ma* Metástasis }\end{array}$ \\
\hline Infecciosas & $\begin{array}{l}\text { Absceso cerebral piógeno, Leucoencefalopatía multi- } \\
\text { focal progresiva* }\end{array}$ \\
\hline $\begin{array}{l}\text { Granulomas } \\
\text { (ver tabla 1) }\end{array}$ & $\begin{array}{l}\text { Infecciosos (micobacterias, hongos)*, parasitarios } \\
\text { (neurocisticercosis, toxoplasmosis* }{ }^{*} \text { tripanosoma*; no } \\
\text { infecciosos (sarcoidosis, cuerpo extraño, otros) }\end{array}$ \\
\hline Otros tumores quísticos & $\begin{array}{l}\text { Quistes: aracnoideo, dermoides, epidermoides, neu- } \\
\text { roentérico, neuroepitelial. Craneofaringioma adamanti- } \\
\text { momatoso, variantes poco frecuentes de meningiomas } \\
\text { y neurofibromas }\end{array}$ \\
\hline Vasculares & Hematomas en reabsorción \\
\hline Post radioterapia & Radionecrosis \\
\hline Desmielinizante & Esclerosis concéntrica de Balo \\
\hline Leyenda: *Más frecuentes en pacientes inmunocomprometidos. \\
\hline
\end{tabular}

den detectarse las hifas o levaduras, según corresponda; la sensibilidad de dicho examen mejora si se practica en tejido fresco, antes de la aplicación de las técnicas de fijación. Además del tratamiento antimicótico sistémico, la resección quirúrgica de las lesiones, siempre que sea posible, es esencial para la resolución de las lesiones.

Las afecciones parasitarias constituyen una causa frecuente de granulomatosis cerebral. La neurocisticercosis es reconocida como la neuroparasitosis más frecuente a nivel mundial y es endémica en América Latina. Se registra con frecuencia como lesiones múltiples que pueden mostrar diferente apariencia imagenológica en relación a la localización, multiplicidad y a la viabilidad del escólex. Se presenta de forma habitual en pacientes inmunocompetentes, procedentes de áreas rurales, con hábitos de ingesta de carne de cerdo sin adecuada cocción y sin fiebre $u$ otros signos sistémicos de infección asociados. Los estudios serológicos como el TSPOT son útiles pero su negatividad no descarta la enfermedad. En pacientes con presentación atípica se requiere de examen histopatológico para asegurar el diagnóstico ${ }^{28}$.

La toxoplasmosis es la parasitosis más frecuente en pacientes inmunocomprometidos, se presentan con lesiones múltiples del encéfalo, con compromiso de ganglios basales. Hay disponibles test serológicos con utilidad limitada para el diagnóstico. En pacientes con 
presentación típica se emplea aún la prueba terapéutica mediante el tratamiento con sulfas o clindamicina que en la mayoría de los casos provoca involución de las lesiones en aproximadamente 6 semanas. En casos dudosos se emplea la biopsia de las lesiones mediante técnicas de mínima invasión, asociado a PCR del tejido biopsiado.

Menos frecuentes son los granulomas causados por amebiasis cerebral, las cuales mantienen una elevada mortalidad. En el examen microscópico del tejido fresco es posible detectar trofozoítos amebianos ${ }^{7,19}$. La Enfermedad de Chagas (trypanosoma cruzi) se mantiene con baja incidencia en Chile desde la erradicación de su transmisión desde hace aproximadamente una década; a diferencia de la toxoplasmosis, las lesiones habitualmente respetan los ganglios basales. En los pacientes inmunodeprimidos, la lesión cerebral es más frecuente que la afectación miopericárdica. Para su diagnóstico se emplea la serología para enfermedad de Chagas, así como PCR o cultivo, en la microscopia directa del LCR pueden detectarse tripomastigotes ${ }^{8}$.

Dentro de los granulomas secundarios a causas no infecciosas o inflamatorias merece la pena comentar, debido a su mayor frecuencia, a la sarcoidosis. Esta es una enfermedad multisistémica, de causa no precisada hasta el momento, que produce granulomas no necróticos, hecho que permite una diferenciación nítida con los tuberculomas en la mayoría de los casos. Más del 90\% de los pacientes evidencia toma pulmonar, mientras que las lesiones extrapulmonares aisladas se detectan solo en el $2 \%$ de los mismos ${ }^{20,25}$.

En la neurosarcoidosis existe una predilección por las leptomeninges de la base craneal y es típica la presentación con disfunción troncular del VII nervio craneano, aunque también pueden ocurrir lesiones de otros nervios craneanos, parenquimatosas, del tallo pituitario e hidrocefalia ${ }^{11}$. Pueden detectarse de manera sincrónica con las lesiones neurológicas linfaadenopatías mediastínicas, eritema nudoso, uveítis, insuficiencia respiratoria y aumento plasmático de los niveles de la enzima convertidora de la angiotensina. El diagnóstico positivo se logra mediante la biopsia ${ }^{9}$.

Dentro del grupo de granulomas de causa no infecciosa se incluyen afecciones menos frecuentes como la en- fermedad de Wegener, la Enfermedad granulomatosa crónica ${ }^{21}$ y la Granulomatosis con poliangeítis ${ }^{6}$.

En el paciente presentado se arribó al diagnóstico de tuberculoma mediante estudio histopatológico. Dicha lesión constituye la forma de presentación más rara de la tuberculosis en el sistema nervioso, representa aproximadamente el $4 \%$ de los tumores intracraneales en países desarrollados, cifra que se eleva hasta un $30 \%$ en los países en vías de desarrollo. La mayoría son lesiones solitarias, aunque se reportan múltiples del 15 al $33 \%$ de los casos, más frecuente en inmunodeprimidos y con tuberculosis pulmonar manifiesta, aunque también se han reportado en individuos sin estas condiciones ${ }^{14}$.

Según Salaskar, et $\mathrm{al}^{22}$ hay varios aspectos que dificultan el diagnóstico inicial de tuberculoma, los cuales confluyeron en el caso presentado: inmunocompetencia del paciente, ausencia de contacto con individuo tuberculoso, tuberculosis pulmonar o signos sistémicos de enfermedad. Por otro lado, también existen condiciones que apoyan este diagnóstico: Granuloma con necrosis caseosa, PCR para micobacterias positivo, cultivo para micobacterium tuberculoso positivo, tinción para bacilo acido alcohol resistente (BARR) del LCR positiva y ADA del LCR mayor a $5,8 \mathrm{u} / \mathrm{l}$.

La historia natural de los tuberculomas cerebrales comprende una mortalidad que supera el $80 \%$ de los casos si no se implementa el tratamiento. Los fármacos antituberculosos constituyen la primera línea terapéutica y pueden complementarse con antiepilépticos y esteroides según la aparición de crisis comiciales y al grado de edema perilesional respectivamente. La resección quirúrgica de la lesión se indica cuando existe importante efecto de masa, mala respuesta al tratamiento farmacológico 0 incertidumbre diagnóstica ${ }^{23}$.

A pesar de no contar con signos patognomónicos, los estudios neuroimagenológicos, sobre todo la RM, son indispensables en el proceso diagnóstico de estos enfermos. Habitualmente se presentan con las llamadas "lesiones en anillo", más evidentes en la secuencia de $T_{1}$ contrastada, las mismas pueden ser detectadas en otros tipos de lesiones: neoplásicas, infecciosas, vasculares, desmielinizantes, entre otras, según se resumen en la Tabla $2^{27}$.
A pesar de lo anterior, algunos signos en la RM se han relacionado en mayor medida con algunas variedades de granulomas intracraneales. Como ya se había comentado, en casos de tuberculoma es más frecuente la detección de lesiones solitarias. Cuando se detecta el "signo de la diana" (centro hipointenso y periferia hiperintensa en $\mathrm{T}_{2}$ ) en lesiones múltiples localizadas en ganglios basales y el tálamo, en pacientes inmunodeprimidos, se sugiere el diagnóstico de toxoplasmosis. En la descripción de dicho signo existe una variedad "excéntrica", evidente en $T_{1}$ contrastada como una pequeña zona nodular, hiperintensa y excéntrica, al interior de una lesión en anillo, y otra "concéntrica", evidente en $T_{2}$ o FLAIR como una zona central hipointensa, con hiperintensidad en la periferia y otra zona hipointensa perimetral a toda la lesión ${ }^{15}$.

Morales et $\mathrm{al}^{17}$, fundamentan que la detección de picos elevados de lípidos en el examen con espectroscopía cerebral por RM, aunque pueden detectarse en gliomas o linfomas, sugiere el diagnóstico de tuberculoma.

Otro aspecto llamativo en el paciente presentado, es la instalación de un síndrome meníngeo larvado acompañado de fiebre, dos semanas después de operado. El paciente se observaba con buen estado general, sin elevación de los parámetros inflamatorios sistémicos y negatividad de los cultivos del LCR. Esta condición, conocida como Meningitis aséptica, puede deberse a variadas causas: infecciones virales (enterovirus, herpes virus, VIH, entre otros), neoplasias cerebrales cuyo contenido irritante se pone en contacto con el LCR (craneofaringioma, tumor epidermoide, tumor dermoide, apoplejía de adenoma hipofisario, entre otras), granulomas cerebrales (por mecanismo similar al de las neoplasias), hemorragia subaracnoidea, administración de fármacos (AINES), causas inflamatorias (Lupus eritematoso sistémico, Enfermedad de Behcet, Sarcoidosis, entre otras), etcétera ${ }^{2,3,4,13,18}$.

Chen et $\mathrm{al}^{5}$, identifican a la meningitis aséptica o química en el 50 al $75 \%$ de todas las meningitis postoperatorias en pacientes neuroquirúrgicos. No obstante, en estos casos siempre es útil la diferenciación con una meningitis bacteriana decapitada, resultado de un tratamiento antimicrobiano ineficiente. En estas circunstancias puede ser útil la 
determinación de lactato en LCR: concentraciones superiores a $6 \mathrm{mmol} / \mathrm{l} \mathrm{se}$ relacionan con meningitis bacteriana, entre 4 y $6 \mathrm{mmol} / \mathrm{l}$ con meningitis bacteriana parcialmente tratada y menores a $2 \mathrm{mml} / \mathrm{l}$ con meningitis aséptica.

A forma se sistematización, varios aspectos deben integrarse para el diagnóstico de las diversas causas de granulomas intracraneales, dentro de ellos resaltan: cuadro clínico neurológico y sistémico, antecedentes de inmunodepresión congénita o adquirida $(\mathrm{VIH} /$ SIDA, cáncer, malnutrición, diabetes mellitus, tratamiento esteroideo crónico o inmunosupresor), contexto epidemiológico, estudios con neuroimágenes y sistémicas (senos perinasales, tórax, abdomen, pelvis, radiografías de miembros), estudios serológicos en plasma y LCR, estudios microbiológicos (directos, cultivos dirigidos a los agentes biológicos más comunes y PCR) del tejido del granuloma fresco e histopatología de las lesiones.

\section{Conclusiones}

Los granulomas intracraneales consti- tuyen un reto diagnóstico. Para el mismo es esencial la sospecha clínica teniendo en cuenta los antecedentes epidemiológicos, la detección de estados de inmunodepresión y de lesiones sistémicas concomitantes. Varios exámenes neuroimagenológicos, inmunológicos y microbiológicos son de ayuda, no obstante el estudio histopatológico de las lesiones es determinante.

Recibido: 17 de marzo de 2018 Aceptado: 02 de junio de 2018

\section{Referencias}

1. Al-Maskari N, Hussain I, Jumara S, Al-Shail A E. Aspergillus flavus-Induced Brain Abscess in an Inmunocompetent Child. Sultan Qaboos University Med J. 2016; 16 (2): 246-49. Doi: 10.18295/SQUMJ.2016.16.02.019.

2. Alonso A, Eisele P, Ebbert DA, Griebe M, Engelhordt B, Szabo K, et al. Leptomeningeal contrast enhacement and blood-CSF barrier dysfunction in aseptic meningitis. Neurol Neuroimmunol Neuroinflamm. 2015; 2: e164-e170. Doi: 10.1212/NXI.0000000000000164.

3. Bahr CN, Boulware RD. Methods of rapid diagnosis for the etiology of meningitis in adults. Biomark. Med. 2014; 8 (9): 1085-1103. Doi: 10.2217/BMM.14.67.

4. Boscolo M, Baleriaux D, Bakoto N, Corvilain B, Devuyst F. Acute aseptic meningitis as the initial presentation of a macroprolactinoma. BMC Research Notes. 2014; 7: 9-13. Doi: 10.1186/1756-0500-7-9.

5. Chen XJ, Alkine CB, Lam CA, Curry TW, Holbrook HE. Aseptic Meningitis with Craniopharyngioma Resection: Consideration after Endoscopic Surgery. J Neurol Surg Rep. 2016; 77: e151-e155. Doi: 10.1055/s-0036-1593470.

6. Costa C, Santiago T, Espírito-Santo J, Rovisco J, Silva J, Malcota A. Pachymeningitis and cerebral granuloma in granulomatosis with polyangiitis: is rituximab a promising treatment option? Acta Reumatol Port. 2017; 42: 82-87.

7. Doan N, Rozansky G, Son N H, Gelsomino M, Shabani S, Mueller W. Granulomatous amebic encephalitis following hematopoietic stem cell transplantation. Surg Neurol Int. 2015 (suppl 18): S459-S462. Doi: 10.4103/2152-7806.166788.

8. Fica A, Salinas M, Jercic MI, Dabanch J, Soto A, Quintanilla S. Enfermedad de Chagas del sistema nervioso central en un paciente con SIDA demostrada por métodos cuantitativos moleculares. Rev Chilena Infectol. 2017; 34 (1): 69-76.

9. Hebel R, Dubaniewicz WM, Dubaniewicz A. Overview of neurosarcoidosis: recent advances. J Neurol. 2015; 262: 258-67. Doi: 10.1007/ s00415-014-7482-9.

10. Hossain C F, Raziul H M, Kabir K S, Morshed A S. Cerebral aspergilloma in a SLE patient: A case report with short literatura review. Asian J Neurosurg. 2014; 9 (2): 58-61. Doi: 10.4103/1793_5482.136710.

11. Imran FT, Nizami S, Eyzner I, Mirani N, Hossain T, Fede R, et al. Vertigo as a Predominant Menifestation of Neurosarcoidosis. Case Reports in Medicine. 2015 (citado el 26/2/18) (aprox. 4 páginas). Doi: 10.1155/2015/397046.

12. Isolan RG, Vieira MD, Hehn F, Antunes MCA. Paracoccidioidomycosis simulating brain tumor. Surg Neurol Int. 2014; 5: 142-46. Doi: $10.4103 / 2152-7806.140656$.

13. Jarrin I, Sellier P, López A, Morgand M, Makovec T, Delcey V, et al. Etiologies and Management of Aseptic Meningitis in Patients Admitted to an Internal Medicine Department. Medicine. 2016; 95 (2): 1-9. Doi: 10.1097/MD.0000000000002372.

14. Kim JK, Jung TY, Lee KH, Kim KS. Radiological Follow-up of a Cerebral Tuberculoma with a Paradoxical Response Mimicking a Brain Tumor. J Korean Neurosurg Soc. 2015; 57 (4): 307-10. Doi: 10.3340/jkns.2015.57.4307.

15. Mahadevan A, Hanumantapura RA, Parthasarathy S, Nath A, Ranga U, Sucarla K. Neuropathological correlate of the "concentric target singn" in MRI of HIV associated cerebral toxoplasmosis. J magn Reson Imaging. 2013; 38 (2): 488-95. Doi: 10.1002/jmri.24036.

16. Monteriro R, Carneiro CJ, Costa C, Duarte R. Cerebral tuberculoma-A clinical Challenge. Respiratory Medicine Case Report. 2013; 9 : 34-37. Doi: 10.1016/j_rmcr.2013.04.003.

17. Morales H, Alfaro D, Mertinot C, Fayed N, Gaskill-Shipley M. MR spectroscopy if intracranial tuberculoma: A singlet peak at 3.8 ppm as potential marker to differentiate them from malignant tumors. Neuroradiol J. 2015; 28 (3): 294-302. Doi: 10.1177/1971400915592077.

18. Mutarelli GE, Adoni T. latrogenic meningitis. Arq Neuropsiquiatr. 2013; 71: 659-60. Doi: 10.1590/0004-282x20130145.

19. Ong TYY, Khan NA, Siddiqui R. Brain-eating amoebae: predilection sites in the brain and disease outcome. J Clin Microbiol. 2017; 55: 1989-97. Doi: 10.1128/JCM.02.300-16.

20. Palmucci S, Torrisi ES, Caltabiano CD, Puglisi S, Lentini V, Grassedonio E, et al. Clinical and radiological festures of extra-pulmonary sarcoidosis: a pictorial essay. Insights Imaging. 2016; 7: 571-87. Doi: 10,1007/s13244-016-0495-4.

21. Roos D. Chronic granulomatous disease. British Medied Bulletin. 2016; 118: 53-66. Doi: 10.1093/bmb/idw009.

22. Salaskar AL, Hassaneen W, Keenan CH, Suki D. Intracranial tuberculoma mimicking brain metástasis. J Can Res Ther. $2015 ; 11: 653-56$. Doi: 10.4103/09731482.138131.

23. Saleh M, Saeedi AA, Pooran AA. Brain Tuberculomas: A Case Report. Jundishapur J Microbiol. 2014 ; 7 (7): e11252. Doi: 10.5812/ jim.11252.

24. Stavrakis C, Narayan A, Varomel O. Cerebral Blastomycosis: Radiologic-Pathologic Correlation of Solitary CNS Blastomycosis Mass-Like Infection. J Clin Imaging Sci. 2015; 5: 30-33. Doi: 10.4103/2156-7514.157854. 
25. Tuna T, Ozkaya S, Drirican A. An intracerebral mass: tuberculosis or sarcoidosis? BMJ Case Report. 2013. (citado el 26/2/18) (aprox. 3 páginas). Doi: 10.1136/bcr-2013-009570.

26. Wagas M, Zafar S, Rehman T, Riyaz M, Bari EM, Idress R. Cerebral aspergilosis and pulmonary tuberculosis in a child with chronic granulomatous disease. Surg Neurol Int. 2016; 7: 62-66. Doi: 10.4103/2152-7806.183166.

27. Yoo-Ri S, Hyeran Y, Sehoon L, Jee-Young K, Suk GH, Kyung SP. Balo's Concentric Sclerosis Mimicking Cerebral tuberculoma. Exp Neuroradiol. 2015; 24 (2): 169-72. Doi: 10.5607/en.2015.24.2.169.

28. Yuzuwa H, Hirose Y, Kimura T, Kimura S, Sugwara H, Yanagisawa A, et al. A case of cerebral tuberculoma mimicking neurocysticercosis. Acute Medicine and Surgery. 2017; 4: 329-33. Doi: 10.1002/cms2.272.

29. Zhou HJ, Zhan RY, Chen MT, Cao F, Zheng JX. Solitary Spinal Dural Syphilis Granuloma Mimicking a Spinal Meningioma. Turkish Neurosurgery. 2014; 24 (2): 288-91. Doi: 10.5137/1019-5149.JTN.5525-11.0.

Correspondencia a:

Dr. Ariel Verela Hernández

varelahernandezariel@gmail.com 